\title{
Erratum
}

\section{Simultaneous excitation-detachment functions for the electron-negative hydrogen ion collisions near the threshold}

\section{P. Grujić, D. Koledin}

Institute of Physics, P.O. Box 57, 68, 11001 Belgrade, Yugoslavia

Z. Phys. D 14, 327-332 (1989)

We have detected an error in Fig. 1. The analytical expression for the detachment function, taken over from [11], $\sigma \sim E^{9 / 4} \exp \{-16.4 \sqrt{E}\}$ should read $\sigma \sim E^{9 / 4} \exp$ $\{-16.4 / \sqrt{E}\}$. The error stems from (28a) in [11], where apparently due to a missprint, sign "-" in front of the exponent " $1 / 2$ " is missing, what can be easily inferred from (27a) from the same reference [cf. also (29)]. As a curve drawn according the correct analytical expression appears practically stepfunction-like (going vertically to infinity), at an arbitrarily chosen point on the abscise, it bears no resemblance to our numerical results, calculated for the excitation-ionization intervals.
Since, however, detachment function from [11] was used only for the sake of comparison, in absence of any other data for the excitation-ionization function, it is of no crucial importance to our calculations and final results and should be ignored in Fig. 1 of our paper. The same holds for the corresponding comments in the text.

We complete the Reference list here:

10. Dimitrijević, M., Grujić, P., Peach, G., Simonović, N.: J. Phys. B23, 1641 (1990)

15. Koledin, D.: J. Phys. B 23, 853 (1990) 